Sharif University of Technology
Scientia Iranica
SCIENTIA

\title{
Investigating the effects of ionic polymer metal composite patches on aeroelastic characteristics of a cantilever wing in supersonic flow
}

\author{
S. Jamshidi, M. Dardel* and M.H. Pashaei \\ Department of Mechanical Engineering, Babol Noshirvani University of Technology, Babol, P.O. Box 484, Iran.
}

Received 4 February 2014; received in revised form 13 November 2014; accepted 23 May 2015

\section{KEYWORDS}

Energy harvesting; IPMC;

Flutter;

Limit cycle;

Supersonic flow.

\begin{abstract}
This work presents energy harvesting from the limit cycle oscillation of low aspect ratio rectangular cantilever wings in supersonic flow. The wing is modeled according to the classical plate theory with von-Karman strain-displacement relations for modeling large deflections due to mid-plane stretching. The aerodynamic pressure is evaluated based on the quasi-steady first-order piston theory. Linear and nonlinear aeroelastic characteristics of the considered model are accurately examined and the effects of Ionic Polymer Metal Composite (IPMC) energy harvesting on flutter margin and limit cycle oscillation amplitudes are investigated. It is shown that the position of IPMC on the wing has a great effect on the amount of harvested power. Since IPMC induces a high level of strain, it produces the static deflection of the wing. This static deflection produces stiffness hardening of the entire system, and, accordingly, can greatly reduce the amplitude of limit cycle oscillation. Obtained results show that the IPMC actuator has more influence on the limit cycle oscillation of the wing, while its effect on flutter instability is negligible.

(C) 2016 Sharif University of Technology. All rights reserved.
\end{abstract}

\section{Introduction}

Research interest in converting ambient vibration energy to usable electrical energy has increased in the last few years. The concept of energy harvesting is particularly useful for wireless sensors powered by batteries, and remotely operated systems with a limited energy source. The goal of the research into vibrationbased energy harvesting is to provide electrical energy for such systems by utilizing the vibrations available in their environment. Already, active materials have been explored as mechanical to electrical energy transducers. Ionic polymers offer several advantages as the choice of active material for the specific application. They have the potential for further development as electromechan-

\footnotetext{
*. Corresponding author.

E-mail address: dardel@nit.ac.ir (M. Dardel)
}

ical transducers. When these polymers are bent, a potential difference between the electrodes is produced. This process is the conversion of mechanical energy to electrical energy. Electrical energy is stored in an electric circuit or consumed.

Electro Active Polymers (EAP) are divided into two categories; electronic, driven by an electric field, and ionic, driven by the diffusion of ions. As stated, the diffusion of ions is the electromechanical coupling in ionic polymers, specifically, the motion of mobile cations. Ionic polymer material has both fixed anions and mobile cations. When the material is hydrated, the cations will diffuse towards an electrode on the material surface under an applied electric field. Inside the polymer structure, anions in the interconnected clusters provide channels for the cations to flow towards the electrode. This motion of ions causes the structure to bend toward the anode [1]. Conversely, bending 
the ionic polymers will force ion diffusion and produce voltage. IPMC consists of a base polymer coated with a metal to act as electrodes.

The electromechanical coupling of EAPs is characterized by high strain, low stress, fast reaction speed, and low drive voltage. Because base material is a polymer, ionic polymer actuation can range to strains of $>10 \%$. EAPs only require a few volts for actuation, usually less than $10 \mathrm{~V}$ [1]. While ionic polymers belong to a larger class of material EAPs, piezoelectric materials are a type of ferroelectric material. Piezoelectric materials have the opposite characteristics of ionic polymers. Piezoelectric materials produce low strain, but large stress. Typical strains for piezoelectric materials are between $0.1 \%$ and $0.3 \%$, while generated stress varies between 30 and $40 \mathrm{MPa}$. Another difference is the drive voltage. Ionic polymers can be activated with low voltages, but piezoelectric materials have actuation values of 50 to $800 \mathrm{~V}$. Therefore, the voltage produced during energy harvesting for ionic polymers is less than piezoelectric materials. Most of the piezoelectric materials used for engineering purposes are ceramic. Because piezoelectric materials are a more rigid structure, they can produce larger forces. Typically, they have an elastic modulus of $50 \mathrm{GPa}$ in the poling direction and $62 \mathrm{GPa}$ in a perpendicular direction, but ionic polymers have an elastic modulus from $150 \mathrm{MPa}$ to $400 \mathrm{MPa}$ [2].

Energy harvesting from base vibration is studied in [3]. Energy harvesting from ambient environments, which is an important source of energy harvesting, is considered in [4]. Fluid-structure interaction is one of these environmental sources. Small diaphragms in pressurized flow channels [5] and large-scale vortex induced vibration based harvesters are other examples of this type [6]. In [7], oscillations induced in a flexible duct wall are considered an energy source. Energy harvesting from low speed flow using small cantilever piezoelectric elements due to external excitation of a gust generator has been studied by [8]. De Marqui et al. investigated harvesting electrical energy from aeroelastic vibrations of the lifting surfaces of UAVs and MAVs, due to their relatively flexible wings [9]. Recently, marine environments have been introduced as a new energy source. These environments, such as beaches and rivers, also show good potential for energy harvesting, so designing an energy harvester for these environments is necessary. Taylor et al. designed an energy harvester that produces power at relatively low flow speeds [10]. In work done by Dunnmon et al., a flexible beam with piezoelectric laminates excited by a uniform axial flow is used as an energy source, whose power is delivered to an electrical impedance load [11].

Aeroelastic systems have different sources of nonlinearity due to their multidisciplinary nature. These nonlinearities may be in the form of structural or aerodynamics types, which result in chaos, limit cycles, and other types of nonlinear behavior. A review of the nonlinear aeroelasticity field and related topics is given in [12]. The main focus of available work on the energy harvesting of aeroelastic systems is on piezoelectric materials. These materials are known to have very good properties and are extensively studied in related literature. In this work, the influence of energy harvesting on the nonlinear aeroelastic response of a cantilevered plate with IPMC laminates is addressed. The nonlinear structural model considered in this work is a nonlinear plate model, based on the classical plate model, with large deflection strains, according to von-Karman theory [13]. These strains are due to in-plane stretching. For aerodynamic modeling, the quasi-steady first-order piston theory is used [14]. At first, modeling of structural, aerodynamics, and ionic polymer metal composites is illustrated. Then, the validity of these equations is shown and their obtained results are presented. Finally, the conclusion to the problem is given.

\section{Theoretical analysis}

The aeroelastic model with attached harvester is shown in Figure 1. The model consists of a cantilevered wing with IPMC laminates attached in a bimorph configuration. The rectangular wing has span, $L$, chord, $c$, and thickness, $h_{p}$. The equations of motion of the wing with embedded IPMC can be derived as follows.

\subsection{Modeling wing in according to classical plate theory}

In classical plate theory, displacement components at a point of height $z$ from the mid-plane of the plate can be expressed as:

$$
\begin{aligned}
& u_{p}(x, y, z, t)=-z \frac{\partial w_{0}(x, y, t)}{\partial x}, \\
& v_{p}(x, y, z, t)=-z \frac{\partial w_{0}(x, y, t)}{\partial y}, \\
& w_{p}(x, y, z, t)=w_{0}(x, y, t),
\end{aligned}
$$

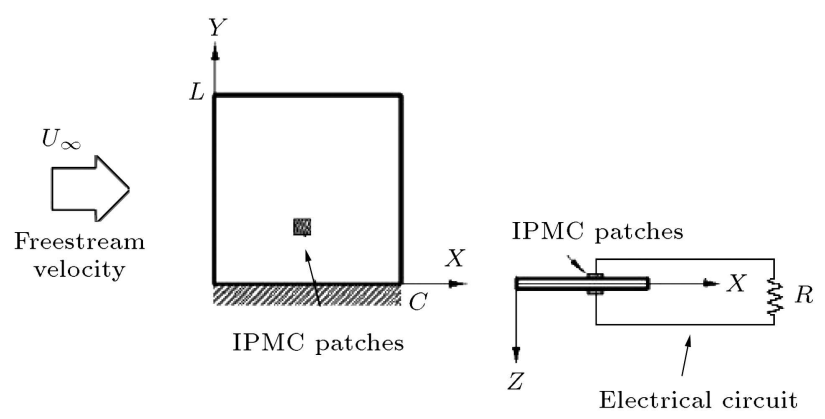

Figure 1. Thin cantilevered wing with embedded IPMC patches and its cross-sectional view. 
where $u_{p}$ and $v_{p}$ denote in-plane, and $w_{p}$ denotes transverse displacements of any point of a cross section of the plate. The subscript ' 0 ' refers to the displacement of a point in a mid-plane. Using von-Karman straindisplacement relations, the nonlinear strain field can be expressed as [15]:

$$
\begin{aligned}
\left\{\begin{array}{c}
\epsilon_{x x} \\
\epsilon_{y y} \\
\gamma_{x y}
\end{array}\right\} & =\left\{\begin{array}{c}
\epsilon_{x x}^{(0)} \\
\epsilon_{y y}^{(0)} \\
\gamma_{x y}^{(0)}
\end{array}\right\}+z\left\{\begin{array}{c}
\epsilon_{x x}^{(1)} \\
\epsilon_{y y}^{(1)} \\
\gamma_{x y}^{(1)}
\end{array}\right\} \\
& =\left\{\begin{array}{c}
\frac{1}{2}\left(\frac{\partial w_{0}}{\partial x}\right)^{2} \\
\frac{1}{2}\left(\frac{\partial w_{0}}{\partial y}\right)^{2} \\
\frac{\partial w_{0}}{\partial x} \frac{\partial w_{0}}{\partial y}
\end{array}\right\}+z\left\{\begin{array}{l}
-\frac{\partial^{2} w_{0}}{\partial x^{2}} \\
-\frac{\partial^{2} w_{0}}{\partial y^{2}} \\
-2 \frac{\partial^{2} w_{0}}{\partial x \partial y}
\end{array}\right\}, \\
\epsilon_{z z}=\epsilon_{x z}=\epsilon_{y z}=0 . &
\end{aligned}
$$

According to the classical plate theory, the strain energy of the plate is given by:

$$
P_{p}=\frac{1}{2} \iiint_{V_{p}}\left[\sigma_{x x} \varepsilon_{x x}+\sigma_{y y} \varepsilon_{y y}+\sigma_{x y} \gamma_{x y}\right] d x d y d z,
$$

where:

$$
\begin{aligned}
\sigma_{x x} & =\frac{E_{p}}{1-\nu_{p}^{2}}\left[\varepsilon_{x x}+\nu_{p} \varepsilon_{y y}\right], \\
\sigma_{y y} & =\frac{E_{p}}{1-\nu_{p}^{2}}\left[\varepsilon_{y y}+\nu_{p} \varepsilon_{x x}\right], \\
\sigma_{x z} & =\frac{E_{p}}{2\left(1+\nu_{p}\right)} \gamma_{x z} .
\end{aligned}
$$

$E_{p}$ and $\nu_{p}$ are Young's module of elasticity and Poisson's ratio of the plate, respectively. By substituting Eq. (4) into Eq. (3), strain energy can be expressed as:

$$
\begin{aligned}
P_{p}= & \frac{E_{p}}{2\left(1-\nu_{p}^{2}\right)} \iiint_{V_{p}}\left[\varepsilon_{x x}^{2}+2 \nu_{p} \varepsilon_{x x} \varepsilon_{y y}+\varepsilon_{y y}^{2}\right. \\
& \left.+\frac{1-\nu_{p}}{2} \gamma_{x y}^{2}\right] d x d y d z .
\end{aligned}
$$

Finally, the strain energy is given by:

$$
\begin{aligned}
P_{p}= & \frac{E_{p}}{2\left(1-\nu_{p}^{2}\right)} \iint\left\{h \left[\frac{1}{4}\left(\frac{\partial w_{0}}{\partial x}\right)^{4}+\frac{1}{4}\left(\frac{\partial w_{0}}{\partial y}\right)^{4}\right.\right. \\
& \left.+\frac{\nu_{p}}{2}\left(\frac{\partial w_{0}}{\partial x}\right)^{2}\left(\frac{\partial w_{0}}{\partial y}\right)^{2}+\frac{1-\nu_{p}}{2}\left(\frac{\partial w_{0}}{\partial x} \frac{\partial w_{0}}{\partial y}\right)^{2}\right] \\
& +\frac{h^{3}}{12}\left[\left(\frac{\partial^{2} w_{0}}{\partial x^{2}}\right)^{2}+2 \nu_{p} \frac{\partial^{2} w_{0}}{\partial x^{2}} \frac{\partial^{2} w_{0}}{\partial y^{2}}+\left(\frac{\partial^{2} w_{0}}{\partial y^{2}}\right)^{2}\right.
\end{aligned}
$$

$$
\left.\left.+2\left(1-\nu_{p}\right)\left(\frac{\partial^{2} w_{0}}{\partial x \partial y}\right)^{2}\right]\right\} d x d y
$$

The kinetic energy of the plate with mass density of $\rho_{p}$ can be expressed as:

$$
T_{p}=\frac{1}{2} \iiint_{V_{p}} \rho_{p}\left[\left(-z \frac{\partial \dot{w}_{0}}{\partial x}\right)^{2}+\left(-z \frac{\partial \dot{w}_{0}}{\partial y}\right)^{2}+\dot{w}_{0}^{2}\right] d x d y d z .
$$

\subsection{Modeling the IPMC patches}

Buechler [16] represented the relation between stress and electric displacement of IPMC materials as follows:

$$
\left[\begin{array}{l}
T \\
D
\end{array}\right]=\left[\begin{array}{cc}
c^{D} & -c^{D} d \\
c^{D} d & \epsilon^{T}
\end{array}\right]\left[\begin{array}{c}
S \\
E_{v}
\end{array}\right]
$$

where $T$ and $S$ represent mechanical stress and strain, $E$ and $D$ denote the electric field and displacement, $d$ is the strain coefficient, $c^{D}$ denotes the stiffness, and superscript $D$ indicates that it is measured under constant electric displacement. $\epsilon^{T}$ is the dielectric permittivity and its superscript, $T$, indicates that it is determined under constant stress. Also, compressed tensor notation, known as Voigt notation, is used due to the symmetry of the strain and stress tensors. These constitutive laws can describe the piezoelectric effect, the driving phenomenon sensors, actuators, and, most recently, energy harvesters. They may be derived directly through a linear electrical enthalpy expression [17]:

$$
H_{i}=\frac{1}{2} T S-\frac{1}{2} E_{v} D
$$

which, considering Eq. (8), can be expanded into the following form:

$$
H_{i}=\frac{1}{2} S c^{D} S-S c^{D} d E_{v}-\frac{1}{2} \epsilon^{T} E_{v}^{2} .
$$

The electromechanical energy within the IPMC laminates is written as defined by the enthalpy function in Eq. (10), and is also integrated over the total volume $\left(V_{i}\right)$ of the laminates as:

$$
H_{i}=\iiint\left(\frac{1}{2} S c^{D} S-S c^{D} d E_{v}-\frac{1}{2} \epsilon^{T} E_{v}^{2}\right) d V_{i} .
$$

The stiffness and strain of IPMC laminates are as follows (with IPMC nonlinearities neglected for simplicity):

$$
c^{D}=\left[\begin{array}{ccc}
\frac{Y}{1-\nu_{i}^{2}} & \frac{\nu_{i} Y}{1-\nu_{i}^{2}} & 0 \\
\frac{\nu_{i} Y}{1-\nu_{i}^{2}} & \frac{Y}{1-\nu_{i}^{2}} & 0 \\
0 & 0 & \frac{Y}{2\left(1+\nu_{i}\right)}
\end{array}\right],
$$




$$
S=\left\{\begin{array}{c}
\epsilon_{x x} \\
\epsilon_{y y} \\
\gamma_{x y}
\end{array}\right\}=z\left\{\begin{array}{c}
-\frac{\partial^{2} w_{0}}{\partial x^{2}} \\
-\frac{\partial^{2} w_{0}}{\partial y^{2}} \\
-2 \frac{\partial^{2} w_{0}}{\partial x \partial y}
\end{array}\right\} .
$$

By substituting Eq. (12) into Eq. (11), enthalpy can be written in the following form:

$$
\begin{aligned}
H_{i}= & \iiint_{V_{p}}\left\{\frac { Y } { 2 } \left[\frac{1}{1+\nu_{i}}\left(z \frac{\partial^{2} w_{0}}{\partial x \partial y}\right)^{2}\right.\right. \\
& +\frac{1}{\nu_{i}^{2}-1}\left[-\left(z \frac{\partial^{2} w_{0}}{\partial x^{2}}\right)^{2}-\left(z \frac{\partial^{2} w_{0}}{\partial y^{2}}\right)^{2}\right. \\
& \left.\left.-2 \nu_{i} z^{2} \frac{\partial^{2} w_{0}}{\partial x^{2}} \frac{\partial^{2} w_{0}}{\partial y^{2}}\right]\right]+\frac{d Y z}{1-\nu_{i}^{2}}\left[\frac{\partial^{2} w_{0}}{\partial x^{2}}\right. \\
& \left.+\frac{\partial^{2} w_{0}}{\partial y^{2}}+\nu_{i} \frac{\partial^{2} w_{0}}{\partial x^{2}}+\nu_{i} \frac{\partial^{2} w_{0}}{\partial y^{2}}\right] E_{v} \\
& \left.-\frac{\epsilon^{T} E_{v}^{2}}{2}\right\} d x d y d z .
\end{aligned}
$$

In addition, the kinetic energy of the IPMC laminates with mass density of $\rho_{i}$ are expressed in the following form:

$$
\begin{aligned}
T_{p}= & \iint_{A_{i}} \rho_{i} H \dot{w}_{0}^{2} d x d y+\rho_{i}\left(H^{3}+\frac{3}{4} h^{2} H+\frac{3}{2} h H^{2}\right) \\
& \iint_{A_{i}}\left[\left(\frac{\partial \dot{w}_{0}}{\partial x}\right)^{2}+\left(\frac{\partial \dot{w}_{0}}{\partial y}\right)^{2}\right] d x d y,
\end{aligned}
$$

where $H$ is the thickness of the IPMC laminate. The electric field in each IPMC laminate, $E_{v}$, is assumed to be uniform throughout the entire laminate and is defined as the voltage potential difference of each laminate divided by the thickness of the laminate $(H)$ :

$$
E_{v}=\frac{v(t)}{H}
$$

\subsection{Equations of motion}

The Lagrangian functional for this wing model with attached harvester is, thus, the difference in kinetic energy and potential energy:

$$
L(w, \dot{w}, v)=K_{i}+K_{p}-P_{p}-H_{i},
$$

where $K_{i}$ and $K_{p}$ denote kinetic energies of the IPMC patches and wing, respectively, $P_{p}$ denotes the strain energy of the wing, and $H_{i}$ denotes bending enthalpy for electrically active patches.
The equations of motion are obtained using the Rayleigh-Ritz method and Lagrange equation. The Rayleigh-Ritz method consists of assuming the form of the solution in terms of admissible functions and generalized coordinates. The required admissible functions must satisfy the geometric boundary conditions of the plate. The equation of motion is written in dimensionless form with the aid of the following quantities:

$$
\begin{aligned}
& \bar{H}=\frac{H}{h_{p}}, \quad \bar{w}_{m n}=\frac{w_{m n}}{h_{p}}, \quad \bar{x}=\frac{x}{c}, \quad \bar{y}=\frac{y}{L}, \\
& D_{p}=\frac{E_{p} h_{p}^{3}}{12\left(1-\nu_{p}^{2}\right)}, \quad \omega_{0}=\sqrt{\frac{D_{p}}{\rho_{p} h_{p} c^{4}}}, \quad \tau=\omega_{0} t
\end{aligned}
$$

where $D_{p}$ and $\omega_{0}$ denote bending rigidity and convenient reference frequency, respectively. Using separation techniques, $w_{0}$ can be expressed as:

$$
w_{o}(\bar{x}, \bar{y}, \tau)=\sum_{m} \sum_{n} \bar{w}_{m n}(\tau) w_{m}(\bar{x}) w_{n}(\bar{y})
$$

\subsubsection{Modeling aerodynamics force}

The aerodynamic pressure at supersonic can be evaluated based on the quasi-steady first-order piston theory [13]:

$$
\begin{aligned}
P_{a}(x, y, t) & \\
& =-\frac{\rho_{0} V_{\infty}^{2}}{\sqrt{M_{\infty}^{2}-1}}\left\{\frac{\partial w_{0}}{\partial x}+\left(\frac{M_{\infty}^{2}-2}{M_{\infty}^{2}-1}\right) \frac{1}{V_{\infty}} \frac{\partial w_{0}}{\partial t}\right\} \\
& =-\left(\lambda \frac{D_{p}}{a^{3}} \frac{\partial w_{0}}{\partial x}+\frac{D_{p} g_{a}}{w_{0} a^{4}} \frac{\partial w_{0}}{\partial t}\right)
\end{aligned}
$$

where:

$$
\lambda=\frac{\rho_{a} V_{\infty}^{2} a^{3}}{\beta D_{p}}, \quad g_{a}=\frac{\rho_{a} V_{\infty}\left(M_{\infty}^{2}-2\right)}{\beta^{3} \rho_{p} H_{c}},
$$

where $\beta=\sqrt{M_{\infty}-1}$ and $\lambda, D_{p}, g_{a}$ and $\omega_{0}$ denote the non-dimensional aerodynamic pressure, bending rigidity, dimensionless aerodynamic damping parameter, and convenient reference frequency, respectively. For $M_{\infty} \gg 1$, the dimensionless aerodynamic damping is approximated as follows [18]:

$$
g_{a}=\sqrt{\frac{\mu}{M_{\infty}} \lambda}
$$

where $\mu$ is the air-panel mass ratio, and $\mu / M_{\infty}$ is assumed to be 0.01 [13]. Note that, since air flow passes over both sides of the wings, the aerodynamic pressure must be considered twice. The first-order piston theory has good accuracy in the range of $1.8 \ll M_{\infty} \ll 5[19]$. 


\subsubsection{Wing aeroelastic equation of motion}

By applying Lagrange-Euler equations, systems of ordinary differential equations are obtained for modal displacement and voltage coordinates, as follows:

$$
\begin{aligned}
& \frac{d}{d t}\left(\frac{\partial L}{\partial \dot{\bar{w}}}\right)-\frac{\partial L}{\partial \bar{w}}=Q_{1}(t), \\
& \frac{d}{d t}\left(\frac{\partial L}{\partial \dot{v}}\right)-\frac{\partial L}{\partial v}=Q_{2}(t) .
\end{aligned}
$$

By substituting Eq. (16) into Eq. (22), the equation of motion for transverse displacement coordinate and voltage are written in the following forms:

$$
\begin{gathered}
{[M]\left\{\bar{w}^{\prime \prime}\right\}+([K])\{\bar{w}\}-[\theta]\{v\}=-\left[K_{\text {Aero }}\right]\{\bar{w}\}} \\
-\left[C_{\text {Aero }}\right]\left\{\frac{d \bar{w}}{d \tau}\right\}+\{N L\}, \\
{\left[C_{p}\right]\{v\}+[\theta]^{T}\{\bar{w}\}-\left\{Q_{2}\right\}=0,}
\end{gathered}
$$

where $Q_{2}$ is the global vector of electric charge output, and $v$ is the global vector of voltage output. By taking the time derivative of Eq. (23), we have:

$$
\left[C_{p}\right]\{\dot{v}\}+[\theta]^{T}\{\dot{\bar{w}}\}-\left\{\dot{Q}_{2}\right\}=0 .
$$

In this work, it is assumed that the electrical charge generated from the harvester is discharged through a load resistor, having resistance, $R_{L}$. A potential difference is developed between the electrodes on the top and bottom surfaces of each IPMC patch, so, each patch can be considered an internal electrode capacitor, $C_{p}$, in parallel with a current source, $I(t)$, as shown in Figure 2 [20].

The electrical boundary condition due to a resistive load $\left(R_{L}\right)$ is given as follows:

$$
\left\{\dot{Q}_{2}\right\}=-\frac{v}{R_{L}} \text {. }
$$

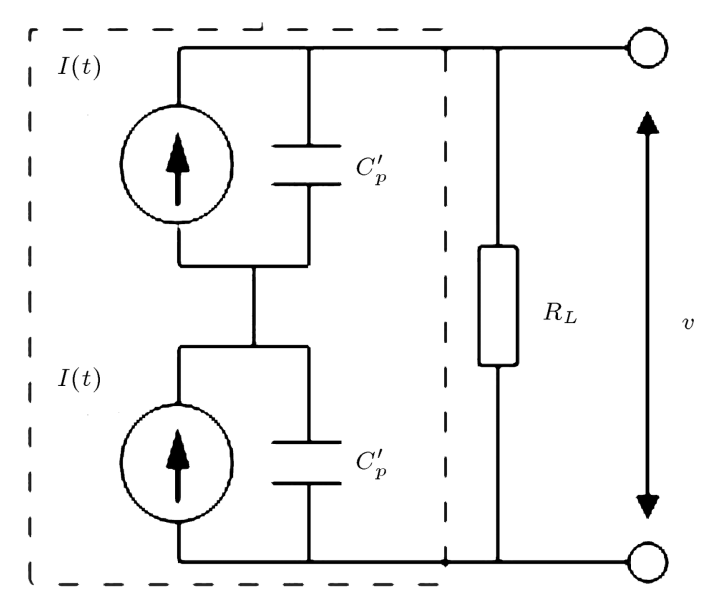

Figure 2. Equivalent circuit for a bimorph energy harvester with series connection for the IPMC patches.
The overall equivalent capacitance, $C_{p}$, and electrical current, $I(t)$, of the bimorph are expressed in the following form:

$$
I(t)=-\theta^{T} \dot{\bar{w}}, \quad C_{p}=\epsilon^{T} \frac{L c}{H},
$$

Using Kirchhoff's law, the governing equation for the electrical circuit, as shown in Figure 3, is given by:

$$
\left[C_{p}\right]\{\dot{v}\}+\left\{\frac{v}{R_{L}}\right\}=-[\theta]^{T}\{\dot{\bar{w}}\} .
$$

For a wing, in accordance with classical plate theory, equation of motion has the following form:

$$
\begin{aligned}
{\left[\begin{array}{cc}
{[M]} & 0 \\
0 & 0
\end{array}\right]\left\{\begin{array}{l}
\bar{w}^{\prime \prime} \\
v^{\prime \prime}
\end{array}\right\}+\left[\begin{array}{cc}
{\left[C_{\text {Aero }}\right]} & 0 \\
{[\theta]^{T}} & {\left[C_{p}\right]}
\end{array}\right]\left\{\begin{array}{l}
\bar{w}^{\prime} \\
v^{\prime}
\end{array}\right\} } \\
+\left[\begin{array}{cc}
{[K]+\left[K_{\text {Aero }}\right]} & -[\theta] \\
0 & \frac{1}{R_{L}}
\end{array}\right]\left\{\begin{array}{l}
\bar{w} \\
v
\end{array}\right\}=\left\{\begin{array}{c}
N L \\
0
\end{array}\right\}_{(28)}
\end{aligned}
$$

The elements of matrices in Eq. (28) are given in Appendix 1. The average power extracted from aeroelastic limit cycle oscillations until time $T$ is given as follows:

$$
\begin{aligned}
P_{\text {total }} & =P_{C}+P_{R}=\frac{1}{T} \int_{0}^{T} \frac{v^{2}}{R}(t) d t+\frac{1}{T} \int_{v_{(0)}}^{v_{(T)}} C_{p} v(t) d v \\
& =\frac{1}{T} \int_{0}^{T} v(t) I(t) d t
\end{aligned}
$$

\subsection{Characterization of IPMC}

The mechanical properties of IPMC are different with usual materials. These properties include the elastic modulus, which has viscoelastic effects, dielectric permittivity, which is not purely capacitive like a piezoelectric device, and the strain coefficient, which again is frequency dependent. These material properties are summarized in Table 1 for the IPMC characteristics given by Buechler [16].

Transfer functions for different material characteristics of IPMC are given in Table 1 [16]. Since these transfer functions have real poles and zeros with large negative values, these poles and zeros are ignored according to the concept of dominate poles and zeros given in automatic control theory. Hence, these properties can be modified. Comparisons between original and simplified models of material transfer functions are plotted and shown in Figure 3 to show the validity of simplifying original transfer functions.

Since IMPC material properties given in Table 1 are frequency dependent, a time response solution of the equation of motion with the current form of 

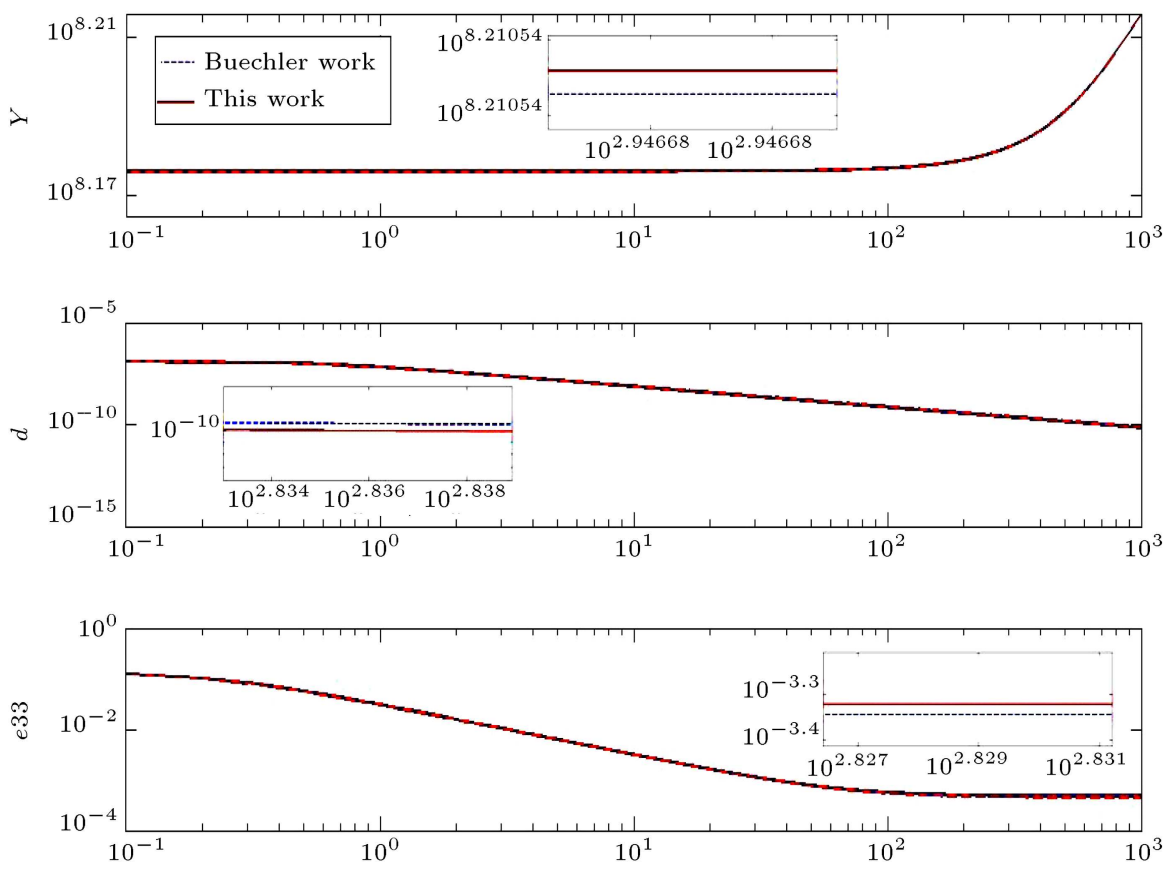

Figure 3. Material characterizations of selected IPMC (Buechler [16] vs. its simplified model).

Table 1. Material properties of selected IPMC.

\begin{tabular}{cll}
\cline { 2 - 3 } & \multicolumn{1}{c}{ Buechler model [16] } & \multicolumn{1}{c}{ Simplified models } \\
\hline $\boldsymbol{d}\left(\frac{\mathrm{m}}{\boldsymbol{v}}\right)$ & $-216 \times 10^{-6} \frac{s+15}{(s+0.6)(s+14)(s+11000)}$ & $-7.3 \times 10^{-8} \frac{1}{s+0.6}$ \\
$\boldsymbol{\epsilon}\left(\frac{\mathbf{F}}{\mathbf{m}}\right)$ & $950 \times 10^{-2} \frac{(s+18.96)(s+69.08)(s+84.48 t)}{(s+0.23)(s+18.83)(s+89.71)(s+10000)}$ & $4.5 \times 10^{-4} \frac{s+69.08}{s+0.23}$ \\
$\boldsymbol{Y}(\mathbf{M P a})$ & $150 \times\left(1+0.22 \frac{s^{2}+12 \times 10^{6} s}{s^{2}+12 \times 10^{6} s+1.44 \times 10^{10}}\right)$ & $50 \times\left(1+0.22 \frac{s}{s+1.2 \times 10^{3}}\right)$ \\
\hline
\end{tabular}

equations of motion is not possible. Hence, it is necessary to include frequency dependent properties directly in the equation of motion. For this purpose, at first, it is necessary to drive the equation of motion in the frequency domain as follows. By taking the Laplace transform of Eq. (28), we have:

$$
\begin{gathered}
\left([M] s^{2}+\left[C_{\text {Aero }}\right] s+\left[K+K_{\text {Aero }}\right]\right)\{\bar{w}(s)\}-[\theta]\{v(s)\} \\
=\{N L(s)\}, \\
s[\theta]^{T}\{\bar{w}(s)\}+\left[C_{p} s+\frac{1}{R_{L}}\right]\{v(s)\}=0 .
\end{gathered}
$$

As seen from Table 1, the transfer function for $d, \epsilon$, and $Y$ have numerator and denominator polynomials in terms of "s". The idea is to transfer Eq. (30) from the frequency domain to the time domain, using the following equality:

$$
s^{n} Q(s)=\frac{\partial^{n} q(t)}{\partial t^{n}} .
$$

By multiplying each equation of Eq. (30) in the common denominator of all terms, and using the idea given by Eq. (31), Eqs. (30) can be written as:

$$
\begin{aligned}
& \sum_{n=0}^{5} c_{n w}^{1}\left\{\frac{\partial^{n} \bar{w}}{\partial \tau^{n}}\right\}-\sum_{n=0}^{2} c_{n v}^{1}\left\{\frac{\partial^{n} v}{\partial \tau^{n}}\right\}=[N L]_{d}, \\
& \sum_{n=0}^{3} c_{n w}^{1}\left\{\frac{\partial^{n} \bar{w}}{\partial \tau^{n}}\right\}+\sum_{n=0}^{4} c_{n v}^{1}\left\{\frac{\partial^{n} v}{\partial \tau^{n}}\right\}=0 .
\end{aligned}
$$

\subsection{Selection of the appropriate mode functions}

According to the Rayleigh-Ritz method, the mode functions necessary to describe different displacements of the problem must satisfy the geometric boundary conditions. Mode functions of a classical plate theory can be obtained from its one dimensional equivalent theory. Hence, for classical plate theory, transverse mode functions of a beam, according to the EulerBernoulli beam, can be used. It is noted that the boundary conditions of the wing are considered to be clamped at one edge and free at three other edges. Hence, mode shapes of the cantilever and free-free beams can be used for this purpose [21]. For the 
free free beam, two rigid modes are necessary to be considered.

Now, after presenting energy harvesting and aeroelastic equations, the results obtained from these equations are given.

\section{Result and discussion}

In this study, the aeroelastic and energy harvesting characteristics of the rectangular wing model of a wing is considered. The plate is considered an aluminum alloy with an aspect ratio of $A R=L / c=1$, stream wise length of $c=0.3 \mathrm{~m}$, thickness of $h=0.001 \mathrm{~m}$, and Poisson's ratio of $\nu=0.3$. For transverse displacement, eight mode numbers are selected; four in streamwise and two in span-wise directions. In the present study, IPMC patches with dimensions of $38 \mathrm{~mm} \times 38 \mathrm{~mm} \times$ $0.3 \mathrm{~mm}$ are considered [16]. $\mathrm{A} \mathrm{Li}+$ cation is the mobile ion and EmI-Tf ionic liquid is the solvent. The electrode is created with $\mathrm{RuO} 2$ and Nafion ${ }^{\mathrm{TM}}$. The outer electrode is gold leaf, which is hot pressed onto the sample. This method of manufacturing was recently developed by Akle, Bennett, and Leo, and it is shown that high strain air-stable actuators and sensors are produced [22]. The selected mechanical and geometrical properties of the considered wing and IPMC models are listed in Table 2.

Typical dimensionless displacements and deflections for the following results are plotted for point $\bar{x}=1$ and $\bar{y}=1$ of the wing.

\subsection{Stability analysis and aeroelastic characteristics of wing without IPMC patches}

At first, the validity of the aeroelasticmodel is studied. To validate the presented equations for a rectangular cantilever wing, LCO amplitude is investigated for two aspect ratios and the results are compared with the solutions given by Weiliang and Dowell [23]. The results presented in [20] are for a wing modeled according to classical plate theory and considering von-Karman strains. It should be mentioned that in Weiliang's work, simulations are carried out for point $\bar{x}=0.75$ and $\bar{y}=1$.

A stability analysis of the aeroelastic system is achieved through an eigenvalue analysis of the linearized system at various aerodynamic pressures. For this purpose, the nonlinear structural terms of Eq. (33) should be linearized at the corresponding equilibrium point. The equilibrium point of the aeroelastic equations can be obtained by equating all time derivatives of the state variable to zero. Then, by determining the Jacobian of the nonlinear structural terms at each equilibrium point, a linearized aeroelastic model will be obtained. These eigenvalues determine the stability of the system. When the real part of one of the eigenvalues becomes positive, the entire system becomes unstable. The dynamic pressure at which purely imaginary eigenvalues occur, and the system is on the verge of instability is considered critical dynamic or flutter pressure and is indicated by $\lambda_{f}$.

At first, the eigenvalue analysis of the wing model for an aspect ratio of 1 is shown in Figure 4 . In this figure, the real part of the eigenvalues of the aeroelastic

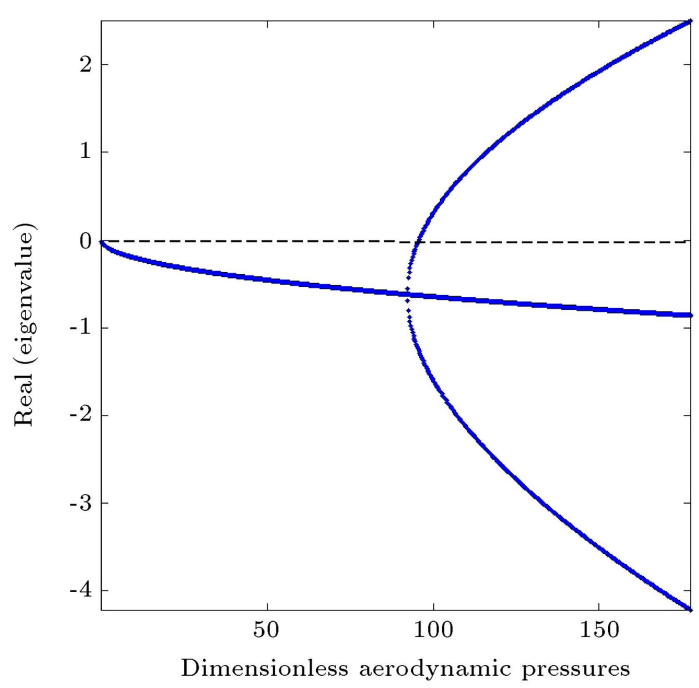

Figure 4. Variation of real part of eignevalues vs. dynamic pressure for aeroelastic wing model with $A R=1$.

Table 2. Mechanical and geometrical properties of IPMC layer and wing.

\begin{tabular}{lcll}
\hline \multicolumn{5}{c}{ IPMC } & & \\
\hline Tensile static modulus, $Y(\mathrm{MPa})$ & 150 & Length, $L_{i}(\mathrm{~mm})$ & 38 \\
Density, $\rho_{i}\left(\mathrm{~kg} / \mathrm{m}^{3}\right)$ & 2600 & Width, $L_{i}(\mathrm{~mm})$ & 38 \\
Poisson's ratio, $\nu_{i}$ & 0.3 & Thickness, $H(\mathrm{~mm})$ & 0.3 \\
\hline Resistance load, $R_{L}$ & $500 \Omega$ & & \\
\hline \multicolumn{4}{c}{ Wing } \\
Tensile static modulus, $E_{p}(\mathrm{GPa})$ & 70 & Length, $L(\mathrm{~mm})$ & 300 \\
Density, $\rho_{p}\left(\mathrm{~kg} / \mathrm{M}^{3}\right)$ & 2700 & Width, $c(\mathrm{~mm})$ & 300 \\
Poisson's ratio, $v_{p}$ & 0.3 & Thickness, $h_{p}(\mathrm{~mm})$ & 1 \\
\hline
\end{tabular}




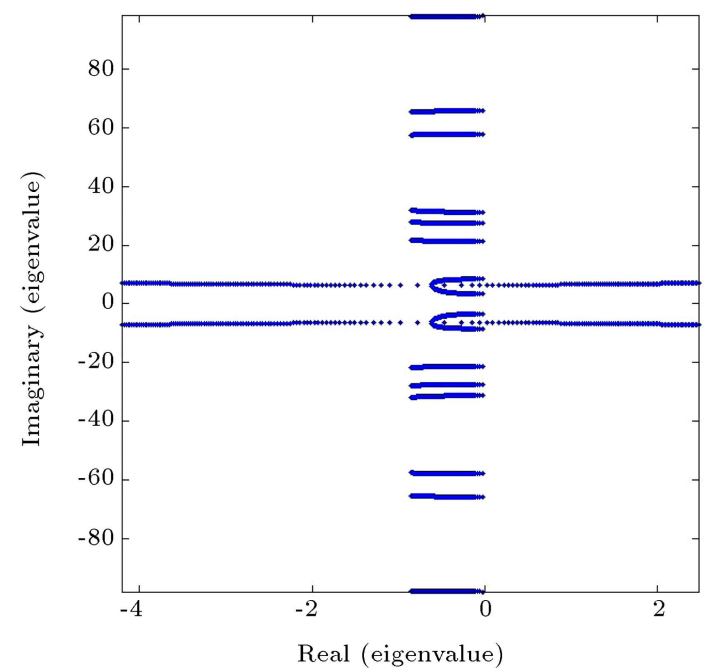

Figure 5. Variation of imaginary part of eignevalues vs. its real part for aeroelastic wing model with $A R=1$.

model vs. dynamic pressures is shown. The real parts of the eigenvalues show the stability of the linearized model of the original nonlinear system. Up to a dynamic pressure of 100 , the system is stable, since all eignevalues have a negative real part. But, at $\lambda_{f}=100$, the first intersection of the eigenvalues branches with the dynamic pressures axis; the related real part is zero and dominant eigenvalues are purely imaginary. The aerodynamic pressure of $\lambda_{f}=100$ shows the critical dimensionless aerodynamic pressure of the wing model and, after that, the system becomes unstable. The calculated critical dynamic pressures for varying aspect ratios are shown in Figure 5. As seen from this figure, there is complete agreement between these results with those given by Weiliang and Dowell [23].

Comparisons of the Limit Cycle Oscillation (LCO) amplitudes for dynamic pressures greater than the critical dynamic pressure of a wing with $A R=1$ is shown in Figure 6 . The obtained results are in good conformity with results presented in [23].

To determine the number of required mode shapes for expressing the transverse displacement of this aeroelastic model, the transverse limit cycle amplitudes of the wing, in terms of different numbers of mode shape, are investigated. From this investigation, eight modes, four in chord-wise and two in span-wise directions, are sufficient for the convergence of the solution.

\subsection{The effect of IPMC patches on natural frequency and stability}

In this section, the effect of attached IPMC patches on the natural frequency and stability of a wing is considered. IPMC patches are attached on the wing in nine positions, according to Figure 7 . It should be noted that each pair of IPMC patches are attached to the wing in a bimorph configuration.

Since the IPMC patches have mass and stiffness,

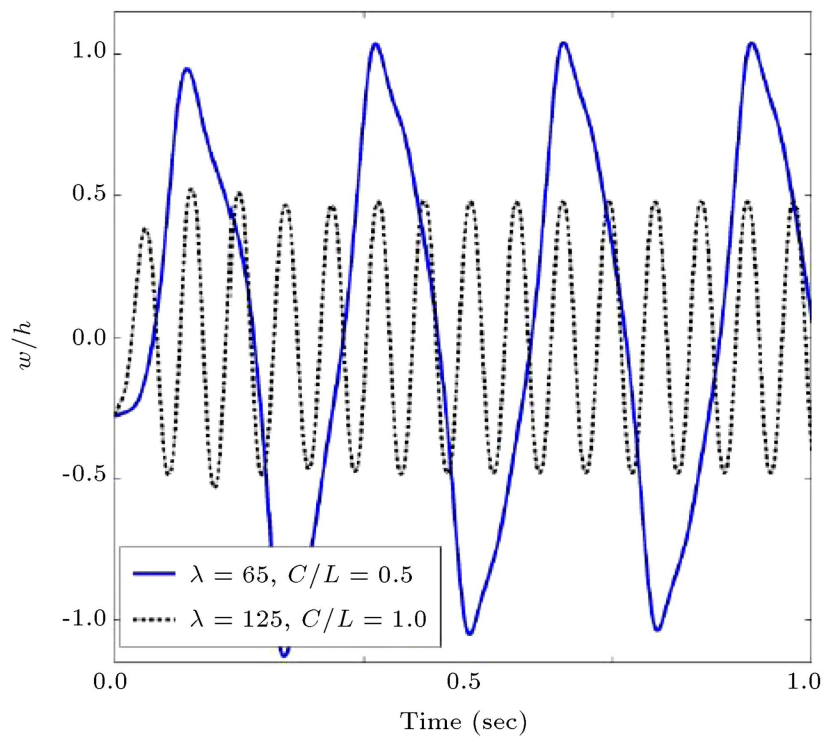

Figure 6. Transverse limit cycle oscillation for two different aluminum wings at different dynamic pressures.

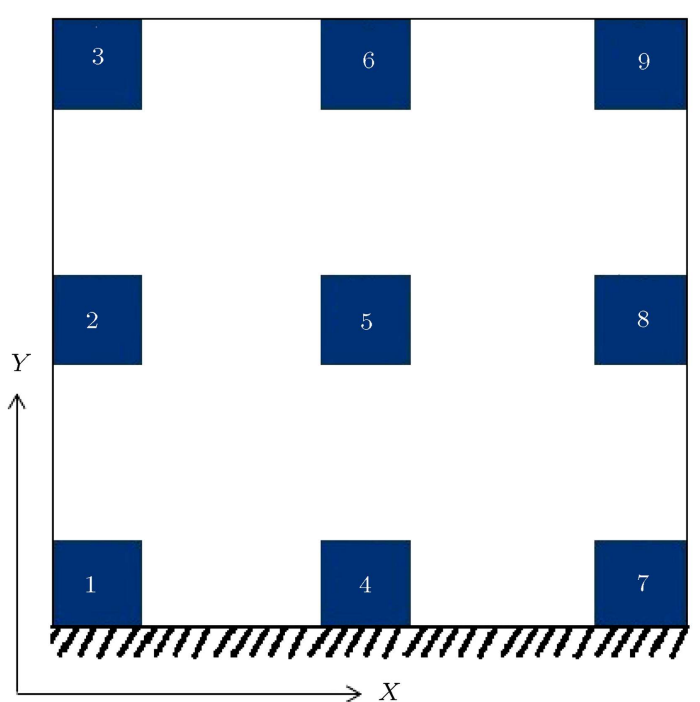

Figure 7. Different locations of embedded IPMCs on the wing.

which influence the natural frequency of the wing, their effect on natural vibration characteristics are first considered and, then, their effects on the stability domain and the flutter margin. The natural frequency of the wing for each pair of IPMC's, according to Figure 7, is shown in Table 3 . In this table, the type of natural frequency, in terms of bending and torsion mode, is also stated. As can be seen, attached IPMC change the natural frequency of the wing. Due to the symmetry, this effect for P1, P2, and P3 is the same as $\mathrm{P} 7, \mathrm{P} 8$ and $\mathrm{P} 9$, respectively. Only at three positions of $\mathrm{P} 1, \mathrm{P} 4$, and $\mathrm{P} 7$, are natural frequencies increased. Torsional frequencies at P2 and $\mathrm{P} 8$ are more reduced than $\mathrm{P} 5$. The general argument regarding the effect of IPMC patches on 
Table 3. Dimensionless natural frequencies for wing with $A R=1$ for different locations of IPMC patches.

\begin{tabular}{|c|c|c|c|c|c|c|c|c|c|c|}
\hline IPMC location $\rightarrow$ & \multirow{2}{*}{$\begin{array}{l}\text { Original } \\
\text { wing }\end{array}$} & \multirow{2}{*}{$\mathbf{P} 1$} & \multirow{2}{*}{ P2 } & \multirow{2}{*}{ P3 } & \multirow{2}{*}{$\mathbf{P} 4$} & \multirow{2}{*}{ P5 } & \multirow{2}{*}{ P6 } & \multirow{2}{*}{ P7 } & \multirow{2}{*}{ P8 } & \multirow{2}{*}{ P9 } \\
\hline Natural frequency $\downarrow$ & & & & & & & & & & \\
\hline$\omega_{1}$ & 3.5036 & 3.5041 & 3.4993 & 3.4517 & 3.5042 & 3.4988 & 3.4509 & 3.5041 & 3.4993 & 3.4517 \\
\hline$\omega_{2}$ & 8.5560 & 8.5574 & 8.5112 & 8.3357 & 8.5561 & 8.5553 & 8.5492 & 8.5574 & 8.5112 & 8.3357 \\
\hline$\omega_{3}$ & 21.5070 & 21.5082 & 21.2285 & 21.4298 & 21.5076 & 21.3917 & 21.1995 & 21.5082 & 21.2285 & 21.4298 \\
\hline$\omega_{4}$ & 27.6255 & 27.6257 & 27.5553 & 26.8078 & 27.6255 & 27.4475 & 27.4387 & 27.6257 & 27.5553 & 26.8078 \\
\hline$\omega_{5}$ & 31.6597 & 31.6583 & 31.1485 & 31.1094 & 31.6598 & 31.6430 & 31.6236 & 31.6583 & 31.1485 & 31.1094 \\
\hline
\end{tabular}

natural frequency characteristics is as follows. At the roots of the wing, i.e. at its clamping point, since the amount of strain is high and its displacement is small, the amount of change in the stiffness of the wing is high, and the change in the mass of the wing is lower than at other positions. Hence, for these locations, natural frequencies will be increased. At the tip of the wing, the displacement is high, but its strain is small due to the free edge boundary conditions. Hence, the equivalent mass of the wing will be increased, with respect to stiffness. Accordingly, the natural frequency will be reduced. With this given description, location 4 has higher equivalent stiffness and lower equivalent mass, with respect to other locations of IPMC.

In Figure 8, critical dynamic pressures of the wing, with $A R=1$, and IPMC locations according to Figure 7, are presented. It can be seen that for position 3 of IPMC, the flutter margin is increased, which is embedded in the upper corner of the leading edge. Embedding IPMC near to the root of the wing (cantilever edge) has little effect on the critical dynamic pressure. IPMC at position 9 has a minimum flutter margin, where IPMC is embedded at the upper corner of the wing. Whenever IPMC's position is closer to

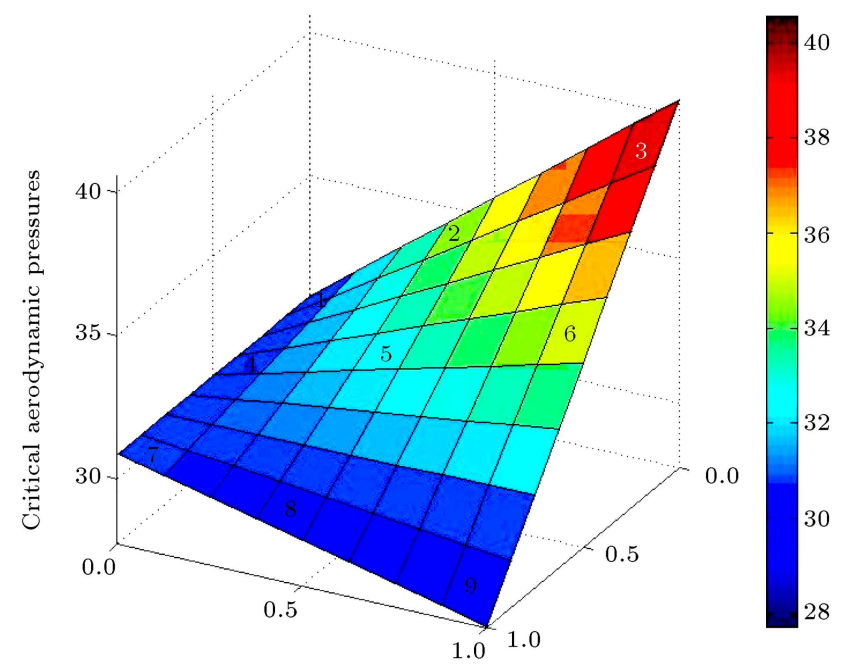

Figure 8. Critical dynamic pressures for wing with $A R=1$ and embedded IPMC at different locations. the upper corner $(\bar{x}=1, \bar{y}=1)$, the flutter margin is further reduced.

The obtained results for critical dynamic pressures are not in conformity with the results obtained from natural frequency examination. This difference is due to the role of the aerodynamics force and the mass and stiffness effects of IPMCs. According to Eqs. (19) and (23), the aerodynamics force induces stiffness and damping forces. These characteristics are dependent on the dynamic pressures. Hence, the aeroelastic characteristics of the wing will vary at different pressures. Dominate eigenvalues of linearized aeroelastic equations versus dynamic pressures are shown in Figure 9. From Figure 10, it is clear that the dominate eigenvalues of the wing at various dynamic pressures are different from the original wing, i.e. at dynamic pressures of zero. In this figure, the lowest frequency is for the first bending mode, whose frequency is nearly constant with the dynamic pressure. The second mode is the torsion mode, whose frequency is continuously reduced with dynamic pressure. The same conditions are presented for two other dominate modes. These results confirm the influence of aerodynamics force on the natural frequencies of the aeroelastic system.

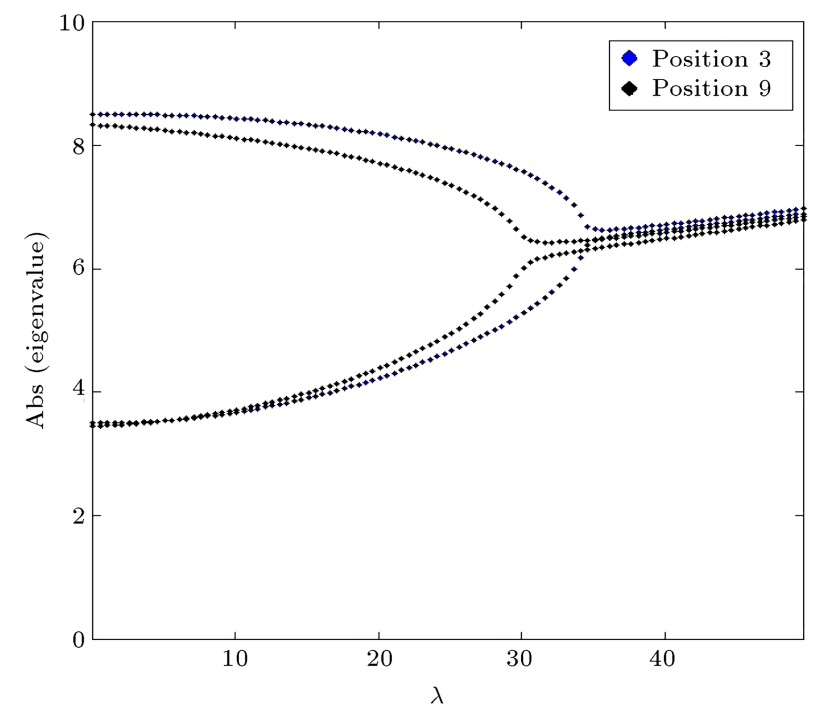

Figure 9. Comparison between flutter margin to IPMC locations of 3 and 9 , for wing with $A R=1$. 


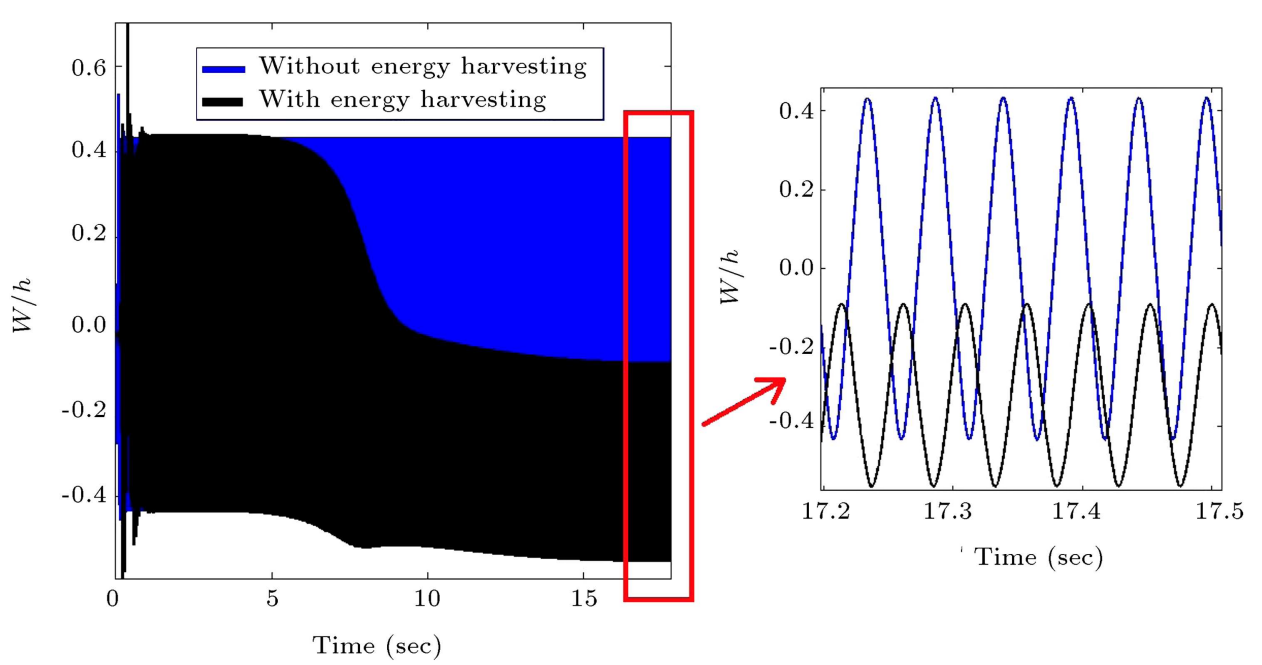

Figure 10. LCO of wings with and without energy harvesting at $\lambda=55$, and IPMC at location of P1.

As seen in a close-up view of Figure 10, the eigenvalues of the wing with IPMC at position 3 are greater than at position 9 . Accordingly, the critical dynamic pressure will be greater, which verifies the result shown in Figure 8. The flutter type for this aeroelastic model is of a bending-torsion type. In this type of flutter, with increasing dynamic pressure, two different modes of the wing, i.e. bending and torsion modes, approach each other. When the distance between these two modes becomes sufficiently small, flutter occurs due to the beating or resonance phenomenon. If two branches of frequencies reach each other at lower dynamic pressure, flutter occurs sooner, and if they reach each other at greater dynamic pressures, flutter occurs later. As seen from Figure 9, for cases 9 and 3, these two branches reach sooner and later than other cases, respectively. Hence, the results given in Figure 8 can be justified.

Now, the effects of energy harvesting on critical dynamic pressure are considered. For this purpose, the real and imaginary parts of the eigenvalues of the linearized aeroelastic model with energy harvesting are shown in Figures 11 and 12, and the obtained results are compared with the case without the harvester. IPMC at location P1 is considered. As seen from this figure, energy harvesting does not change the flutter margin. This means that the stability of the aeroelastic model with and without energy harvesting is nearly the same. The role of energy harvesting will be better studied by investigating the limit cycle oscillation of a wing.

Imaginary versus real parts of the eigenvalues of linearized aeroelastic equations, with and without energy harvesting, for a wing with $A R=1$ and IPMC at position 1, are shown in Figure 12. As seen from this figure, eigenvalues for these two cases are completely the same, and this implies that the damping and frequency of the system for these two cases are not

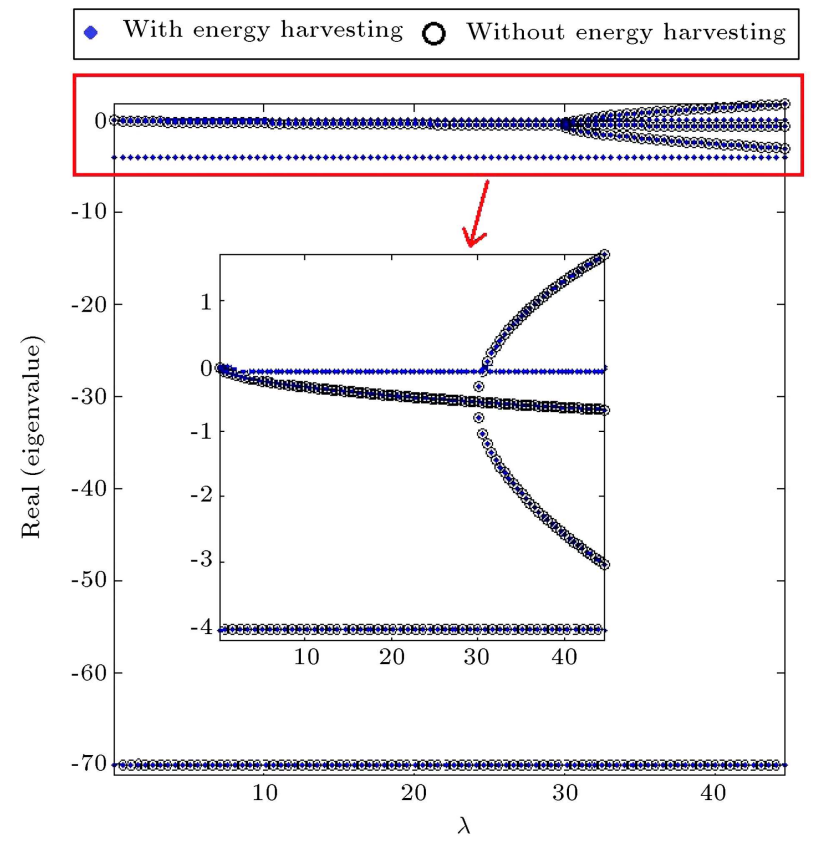

Figure 11. Real part eigenvalues vs. dynamic pressures with and without energy harvesting.

influenced by harvesting the energy. Accordingly, the flutter margin of the aeroelastic system in these two cases is the same.

\subsection{Investigating the effect of energy harvesting on limit cycle oscillations}

In this section, the effect of IPMC patches on the nonlinear behavior of the considered wing model is investigated. Two cases of without and with energy harvesting are studied. For the first case, the effect of structural characteristics of IPMC on nonlinear aeroelastic characteristics, such as Limit Cycle Oscillation (LCO) amplitude, will be studied, and, in the second case, the amount of decrease in LCO amplitude will be considered. 


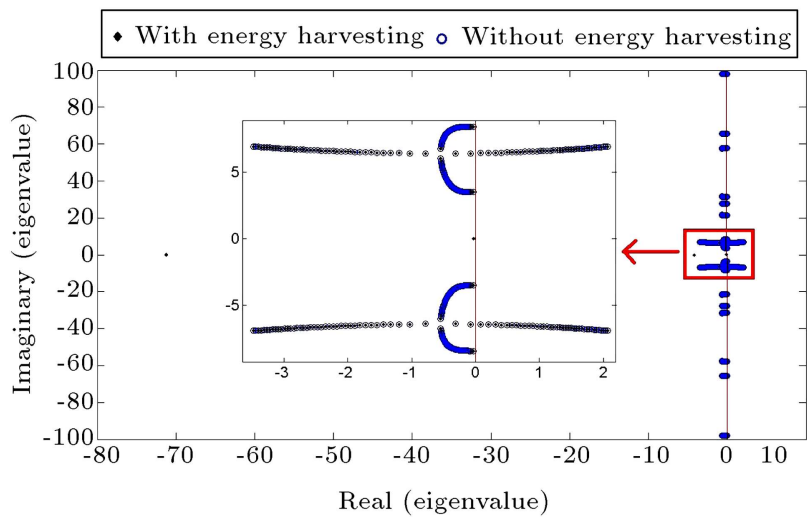

Figure 12. Imaginary vs. real part of eigenvalues of linearized aeroelastic equations with and without energy harvesting for wing with $A R=1$ and IPMC at position 1 .

Dimensionless transverse displacement and limit cycle oscillations, with and without energy harvesting, for a wing with $A R=1$ and IPMC location of 1 at $\lambda=55$ are shown in Figure 10. As seen from this figure, the effect of energy harvesting on reducing the amplitude of the limit cycle oscillation is considerable, although its effect on flutter margin is not.

\subsection{Investigation of the effect of position of \\ IPMC on Limit Cycle Oscillations (LCO)}

Transverse displacements of wing for different positions of IPMC at three dimensionless aerodynamics pressures are shown in Figure 13. As seen from this figure, when energy is harvested from the system, oscillations of the wing are around a non-zero equilibrium point. In other words, the wing has a static deflection. Also, as seen from this figure, the amount of reduction in LCO amplitude, due to energy harvesting through IPMC patches, is considerable, especially at higher dynamics pressures.

Now, there is an important question. While energy harvesting does not have a considerable effect on the flutter boundary, how is this amplitude reduction in limit cycle oscillation justified? Figures 10 and 13 show that when energy is extracted from wing oscillations, a static deflection is produced. Considering that the IPMC has a high level of strain, the resulting static deflection can be considerable. Static deflection results in nonlinear stiffness terms that have an odd degree (third for single mode, and higher for multiple mode), and produces stiffness hardening. As a result, the stiffness of the entire system increases and the amplitude of oscillations is reduced.

\subsection{Investigation of the effect of IPMC patch's position on the harvested power}

The amount of power produced by IPMC patches for the wing with $A R=1$ is shown in Figures 14 . Mean output power is calculated as $P(T)=\frac{1}{T} \int_{0}^{T} v(t) I(t) d t$. As seen from Figure 14, for this dynamic pressure, the maximum harvested power is about $0.17 \mathrm{~mW}$ at $\lambda=$ 55 , which is related to the IPMC at position 4 .
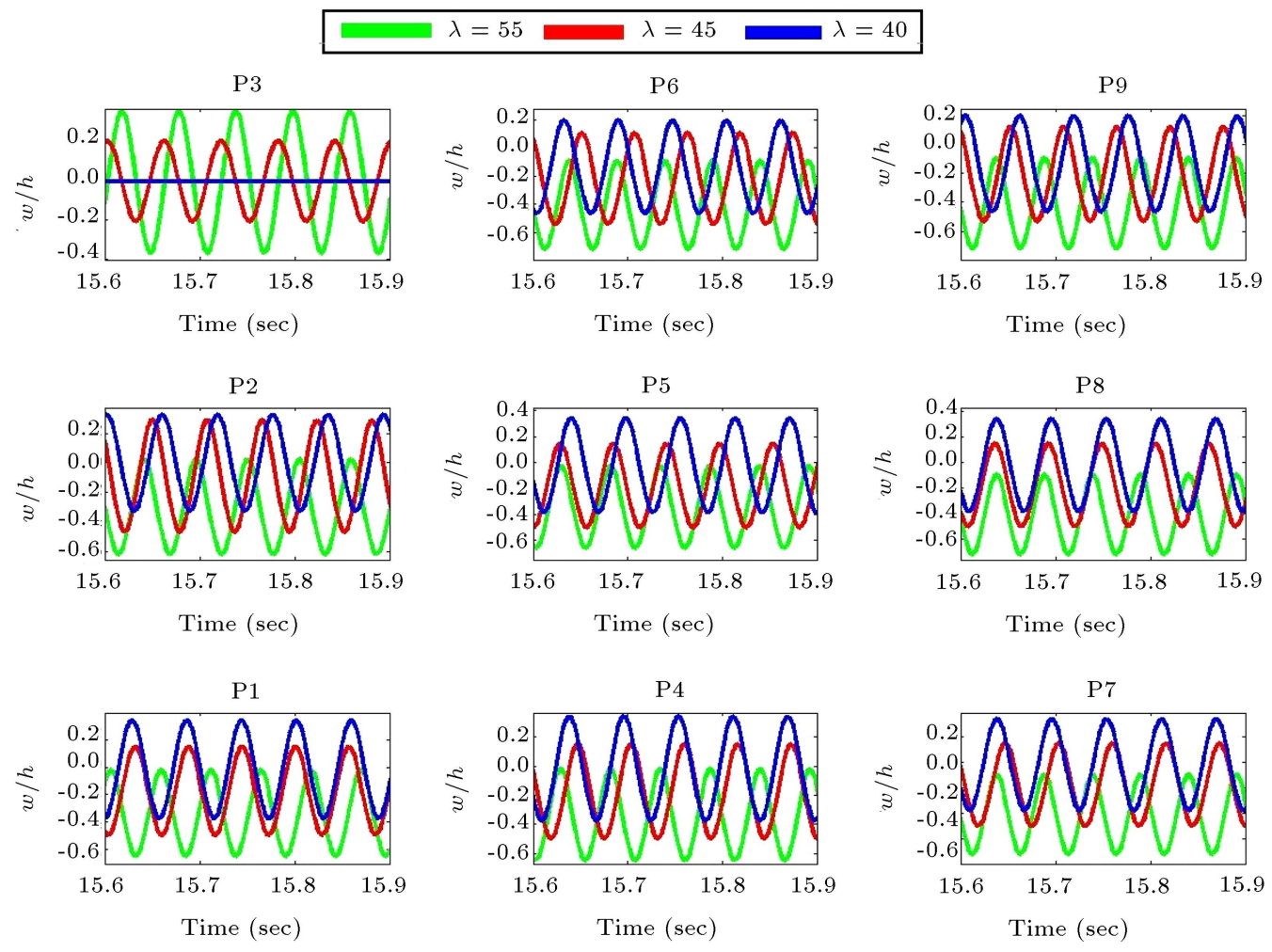

Figure 13. LCO of wing with energy harvesting for all locations of IPMC at $\lambda=40,45,55$. 


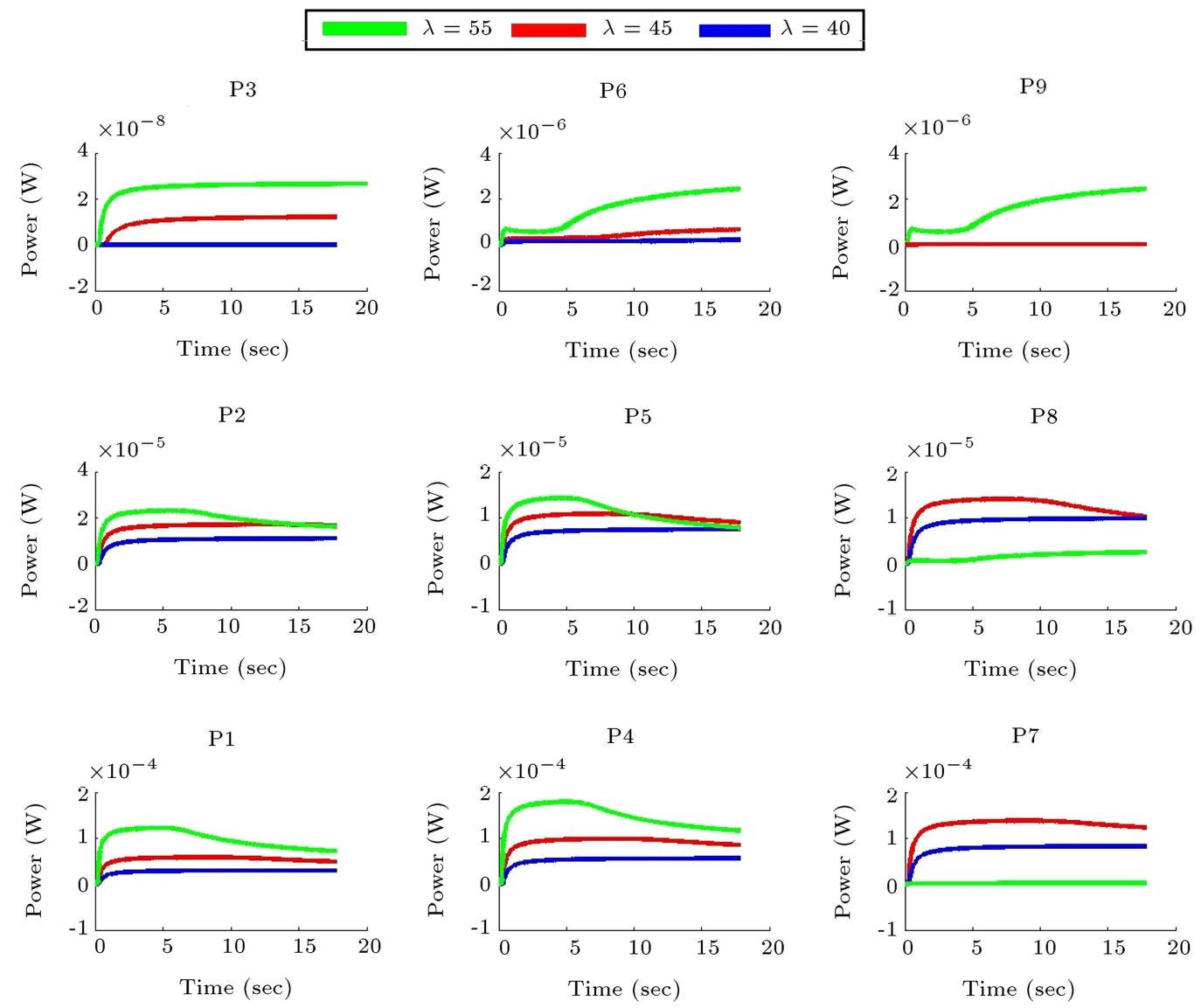

Figure 14. Power produced from LCO for nine locations of IPMC at $\lambda=40,45,50$.

\section{Conclusion}

In this work, energy harvesting from an aeroelastic system by ionic polymer metal composites in supersonic flow is studied. The aerodynamic pressure is evaluated based on the quasi-steady first-order piston theory. Characteristics of the IPMC depend on frequency. They typically cannot be modeled in the time domain. Hence, by proposing a new idea, electromechanical equations are transferred into the time domain. The effects of the mass and stiffness of the polymer embedded on the wing were also studied. Whenever IPMC is embedded closer to the cantilever edge of the wing, the power delivered to the electric circuit becomes greater. At higher dynamic pressure, the harvested power increases. Energy harvesting does not change the flutter margin. When energy is harvested, the wing has a static deflection. Considering that the IPMC has a high level of strain, the resulting static deflection can be considerable. Nonlinear hardening stiffness is resulted from this static deflection. Accordingly, the total stiffness of the entire system will be increased and the amplitude of oscillations will be reduced. This is the reason for the high amount of reduction in the amplitude of limit cycle oscillations.

\section{References}

1. Bar-Cohen, Y., Electroactive Polymer (EAP) Actuators as Artificial Muscles Reality, Potential, and Challenges, SPIE Press, Bellingham, WA (2001).

2. Leo, D.J. "Active materials and smart structures - I", Course Notes, Virginia Tech course ME 5984 (2003).

3. Sodano, H., Inman, D.J. and Park, G. "A review of power harvesting from vibration using piezoelectric materials", The Shock and Vibration Digest, 36, pp. 197-205 (2004).

4. Eichorn C., Goldschmidtboeing, F. and Woias, P. "A frequency tunable piezoelectric energy converter based on a cantilever beam", In Proceedings of Power MEMS 2008 Micro EMS2008, pp. 309-312 (2008).

5. St Clair, D., Bibo, A., Sennakesavababu, V.R., Daqaq, M.F. and Li, G. "A scalable concept for micropower generation using flow-induced self-excited oscillations", Applied Physics Letters, 96, pp. 144103-1144103-3 (2010).

6. Bernitsas, M., Raghavan, K., Ben-Simon, Y. and Garcia, E. "VIVACE (Vortex Induced Vibration Aquatic Clean Energy): a new concept in generation of clean and renewable energy from fluid flow", Journal of Offshore Mechanics and Arctic Engineering, 130, pp. 041101-1-041101-15 (2008). 
7. Akaydin, H.D., Elvin, N. and Andreopoulos, Y. "Wake of a cylinder: paradigm for energy harvesting with piezoelectric materials", Experimental Fluids, 49, pp. 291-304 (2009).

8. Erturk, A., Vieira, W.G.R., DeMarqui, C. and Inman, D.J. "On the energy harvesting potential of piezoaeroelastic systems", Applied Physics Letters, 96, pp. 184103-1-184103-3 (2010).

9. De Marqui, C., Erturk, A. and Inman, D.J. "Piezoaeroelastic modeling and analysis a generator wing with continuous and segmented electrodes", Journal of Intelligent Material Systems and Structures, 21, pp. 983-993 (2010).

10. Taylor, G.W., Burns, J.R., Kamann, S.M., Power, W.B. and Welsh, T.R. "The energy harvesting Eel: a small subsurface ocean/river power generator", IEEE Journal of Oceanic Engineering, 26, pp. 539-547 (2001).

11. Dunnmon, J.A., Stanton, S.C., Mann, B.P. and Dowell, E.H. "Power extraction from aeroelastic limit cycle oscillations", Journal of Fluids and Structures, 27(8), pp. 1182-1198 (Nov. 2011) ISSN 0889-9746, http://dx.doi.org/10.1016/j.jfluidstructs.2011.02.003.

12. Dowell, E.H., Edwards, J. and Strganac, T.W. "Nonlinear aeroelasticity", J. Aircraft, 40(5), pp. 857-874 (2003).

13. Dowell, E.H., Aeroelasticity of Wings and Shells, Noordhoff International Publishing Company, Liyden, pp. 1-9, 19-26 (1975).

14. Hall, K.C. "Eigenanalysis of unsteady flows about airfoils, cascades, and wings", AIAA J., 32(12), pp. 2426-2432 (1994).

15. Reddy, J.N., Mechanics of Laminated Composite Wings and Shells: Theory and Analysis, Second Ed., CRC Press, Boca Raton (2003).

16. Bucchler, M.A. "Variational modeling of ionic polymer-based structures", MS Thesis, Virginia Polytechnic Institute and State University (2005).

17. Samuel, C. "Stanton, nonlinear electroelastic dynamical systems for inertial power generation", $\mathrm{PhD}$ Thesis, Department of Mechanical Engineering and Materials Science, Duke University (2011).
18. Xue, D.Y. "Finite element frequency domain solution of nonlinear panel flutter with temperature effects and fatigue life analysis", PhD Dissertation, Engineering Mechanics, Old Dominion University, Norfolk, VA (1991).

19. Vedeneev, V.V. "Panel flutter at low supersonic speeds", J. Fluid Structure, 29, pp. 79-96Y (2012).

20. Weiliang, Y.E. and Dowell, E.H. "Limit cycle oscillation of a fluttering cantilever plate", AIAA Journal, 29(11), pp. 1929-1936 (1991).

21. Preumont, A., Dynamics of Electromechanical and Piezoelectric Systems, Springer, Brussels (2006).

22. Rao, S.S., Vibration of Continuous Systems, Wiley, New Jersey (2007).

23. Akle, B.J., Bennett, M.D. and Leo, D.J. "Highstrain ionomeric-ionic liquid composites via electrode tailoring", Proceedings of IMECE, No. 61246 (2004).

\section{Biographies}

Shahab Jamshidi received BS and MS degrees in Engineering Mechanics, in 2011 and 2013, respectively, from Babol University of Technology, Iran. His research interests include mechanical nonlinear vibrations and dynamics, smart structures, energy harvesting, aeroelasticity, and liquid sloshing ynamics.

Morteza Dardel received a PhD degree from Amirkabir University, Iran, in Solid Mechanics, in 2009, and is currently Assistant Professor in the Faculty of Mechanical Engineering at Babol Noushirvani University of Technology, Iran. His research interests are aeroelasticity, nonlinear dynamics, vibration, and control of continuous systems and smart structures.

Mohammad Hadi Pashaei received a $\mathrm{PhD}$ degree in Space Structures from the University of Surrey, UK, in 2004, and is currently Assistant Professor in the Faculty of Mechanical Engineering at Babol Noushirvani University of Technology, Iran.. His research interests include structural dynamics, damping in structures, and vibrational systems analysis. 